\title{
EFFECT OF THREE EMERGENT MACROPHYTE SPECIES ON NUTRIENT RETENTION IN AQUATIC ENVIRONMENTS UNDER EXCESS NUTRIENT LOADING
}

Myrto Nikolakopoulou, Alba Argerich, Susana Bernal, Esperança Gacia, Miquel Ribot, Eugènia Martí, Albert Sorolla, and Francesc Sabater

The document contains one page with one Table (S1). 
Table S1. Chemical properties of outflow water in the flumes for each treatment during the experiment $^{\mathrm{a}}$

\begin{tabular}{|c|c|c|c|c|c|}
\hline \multirow{6}{*}{ 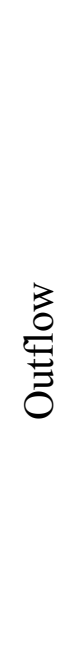 } & Variable & Control & Iris & Phragmites & Schoenoplectus \\
\hline & $\mathrm{NH}_{4}{ }^{+}\left(\mathrm{mg} \mathrm{N} \mathrm{L}^{-1}\right)$ & $0.36 \pm 0.16$ & $0.30 \pm 0.14$ & $0.03 \pm 0.00$ & $0.10 \pm 0.04$ \\
\hline & $\mathrm{NO}_{3}^{-}+\mathrm{NO}_{2}^{-}\left(\mathrm{mg} \mathrm{N} \mathrm{L}^{-1}\right)$ & $4.96 \pm 1.21$ & $2.41 \pm 0.71$ & $1.50 \pm 0.09$ & $4.21 \pm 1.22$ \\
\hline & SRP $\left(m g P^{-1}\right)$ & $0.65 \pm 0.14$ & $0.11 \pm 0.01$ & $0.21 \pm 0.02$ & $0.53 \pm 0.18$ \\
\hline & $\operatorname{DON}\left(\mathrm{mg} \mathrm{N} \mathrm{L}^{-1}\right)$ & $2.42 \pm 0.97$ & $4.35 \pm 0.63$ & $1.47 \pm 0.08$ & $4.2 \pm 1.33$ \\
\hline & TDN $\left(m g ~^{-1}\right)$ & $7.74 \pm 1.24$ & $7.06 \pm 0.88$ & $2.99 \pm 0.07$ & $8.50 \pm 0.91$ \\
\hline
\end{tabular}

a Values are means \pm standard error for the different days that each treatment (Control, Iris, Phragmites, Schoenoplectus) was sampled. Data of $\mathrm{NH}_{4}^{+}, \mathrm{NO}_{3}{ }^{-}+\mathrm{NO}_{2}^{-}$, SRP, and TDN was averaged from two inflow water measurements per flume $(n=6$ per treatment), during the longitudinal sampling. Data presented here are not to be compared with $\mathrm{NH}_{4}{ }^{+}, \mathrm{NO}_{3}{ }^{-}+\mathrm{NO}_{2}{ }^{-}$and SRP inflow concentrations presented in Table 2 of the manuscript, since they derive from different samplings. DON was estimated as TDN - DIN. 